\title{
Development of a novel, accurate, automated, rapid, high-throughput technique suitable for population-based carrier screening for Fragile $\mathrm{X}$ syndrome
}

Charles M. Strom, MD, $P h D^{1}$, Donghui Huang, PhD $D^{1}$, Yuanyin Li, PhD $D^{1}$, Feras M. Hantash, PhD ${ }^{1}$, Jenny Rooke, PhD ${ }^{2}$, Steven J. Potts, $P h D^{1}$, and Weimin Sun, $P h D^{1}$

\begin{abstract}
Purpose: To develop a high-throughput, automated, accurate method suitable for population-based carrier detection of fragile $X$ syndrome. Methods: We developed a new method called capillary Southern analysis that allows automated high-throughput screening for expanded fragile $\mathrm{X}$ mental retardation 1 (FMR1) alleles. Initially samples are analyzed by a multiplex polymerase chain reaction that contains an internal control to establish gender. All females heterozygous for two normal alleles are reported as normal without further analysis. All females homozygous at the FMR1 locus (24\% of all analysis) are then analyzed by capillary Southern analysis. Theoretically this method can detect expansion as high as 2000 CGG repeats, although in our series the largest nonmosaic FMR1 present was 950 CGG repeats. After assay development, we performed capillary Southern analysis on 995 female and 557 male samples submitted for fragile $X$ syndrome testing by polymerase chain reaction and Southern blot. Results: The polymerase chain reaction/capillary Southern analysis technique identified $100 \%$ of six female premutation carriers, seven full mutation carrier females, one premutation male, and five affected males. There was only one discrepancy between analysis by polymerase chain reaction/Southern blot and analysis by polymerase chain reaction/capillary Southern analysis. A single female sample appeared to be heterozygous for a premutation allele by polymerase chain reaction/capillary Southern analysis but was negative by Southern blot. It is possible this patient is a mosaic for the premutation allele, but because samples were deidentified, we were unable to determine whether this was a true false positive. Conclusion: We have developed an automated, high-throughput technique capable of detecting carriers of fragile $X$ syndrome with $100 \%$ sensitivity and at least 99.5\% specificity. This should allow population-based carrier detection for the most commonly inherited form of mental retardation. Genet Med 2007:9(4):199-207.
\end{abstract}

Key Words: Fragile $X$ syndrome, carrier screening

Fragile X syndrome (FXS) is the most commonly inherited form of mental retardation, second only to Down syndrome as the most frequent genetic cause of mental retardation. FXS is caused by mutations in the FMR1 gene coding for the fragile $\mathrm{X}$ mental retardation protein (FMRP). ${ }^{1}$ There is no agreement on the incidence of FXS in various populations. Estimates for white populations vary from 1:3717 to 1:8918 males; studies of populations of African ancestry have estimated higher frequencies of approximately 1:2500 males. $^{2}$

\footnotetext{
From the ${ }^{1}$ Quest Diagnostics, Nichols Institute, San Juan Capistrano, California; ${ }^{2}$ Fidelity Biosciences, Cambridge, Massachusetts.

Charles M. Strom, MD, PhD, Quest Diagnostics, Nichols Institute, 33608 Ortega Highway, San Juan Capistrano, CA 92690; E-mail: charles.m.strom@questdiagnostics.com

The authors declare no conflict of interest.

Submitted for publication November 30, 2006.

Accepted for publication January 24, 2007.

DOI: 10.1097/GIM.0b013e31803d3ac9
}

FXS is inherited as an X-linked dominant disease. Males have mental retardation with an average IQ $<50$, attentiondeficit/hyperactivity disorder, and variable physical features such as post pubertal macroorchidism and dysmorphic cranial features. ${ }^{1}$ The symptoms of FXS in females are usually milder than those in males and include attention deficit disorder, learning disabilities, and personality disorders. Couples with affected children face significant family, medical, emotional, educational, psychological, and financial challenges. Children with FXS require medication and special education services, and adult males with FXS are usually incapable of independent living.

Almost all cases of FXS are due to an expansion of a CGG tandem repeat in the $5^{\prime}$ untranslated region of the FMR1 gene to $>200$ repeats. This expansion leads to methylation of the $5^{\prime}$ untranslated region causing decreased expression of FMR1. Rarely, FXS is due to point mutations or complete deletions of the FMR1 gene. ${ }^{3-8}$ 


\section{Strom et al.}

The inheritance patterns in FXS are complex. The American College of Medical Genetics classifies normal FMR1 alleles as CGG repeats of $<45$. Premutation alleles consist of 55-200 CGG repeats, and full mutation alleles have $>200$ repeats. Alleles between 45 and 55 are considered indeterminate or "gray zone" alleles. ${ }^{9}$

When inherited through the mother, premutation alleles may expand to larger premutation alleles or to full mutation alleles. ${ }^{10}$ Therefore, women who are carriers of either premutation or full mutation alleles are at risk of conceiving affected children. Female premutation carriers have no mental dysfunction due to their FMR1 alleles, but $20 \%$ will experience premature ovarian failure, defined as menopause younger than 40 years of age. ${ }^{11,12}$ In contrast, premutation alleles, when inherited through the father, do not expand, but all his daughters will be at risk of having affected children. Approximately $40 \%$ of males with premutation alleles will suffer from fragile X tremor ataxia syndrome (FXTAS) after the age of 50 years. ${ }^{13}$ Recently, there has been a report of FXTAS in premutation carrier females. ${ }^{14}$ Although "gray zone" alleles (45-55 CGG repeats) are considered above the normal range, there have been no reported instances of an allele of $<56$ repeats expanding to a full allele in a single meiosis. ${ }^{10}$

Carrier detection pilot studies in various populations have been performed. The observed carrier frequencies varied: 1:70 in Britain, ${ }^{15}$ 1:113 in Israel, ${ }^{16}$ and 1:259 in Quebec. ${ }^{17}$ Population-based carrier screening has been implemented in the United States for cystic fibrosis in Caucasians (carrier frequency 1:28), ${ }^{18,19}$ and Tay-Sachs disease (carrier frequency 1:30), Canavan disease (carrier frequency 1:45), and familial dysautonomia (carrier frequency 1:42) in Ashkenazi Jewish (AJ) populations. ${ }^{20}$ These disorders are autosomal recessive diseases, and therefore both parents must be carriers for a pregnancy to be at risk of an affected fetus. In contrast, FXS is an $\mathrm{X}$-linked disorder. When a woman is a premutation or full mutation carrier, all her pregnancies are at risk. Although in premutation carriers, the amount of risk will increase with increasing numbers of CGG repeats, there will be at least some increased risk for all her pregnancies. Therefore, populationbased carrier screening for FXS would have a higher yield of identifying at-risk couples (1:70-1:259) than cystic fibrosis in Caucasian populations (1726:1764), or the three disorders in AJ populations: Tay-Sachs disease (1:900), Canavan disease (1:2025), and familial dysautonomia (1:764), for which population-based carrier screening has already been implemented.

A financial analysis revealed population-based FXS carrier detection would be cost-effective. ${ }^{21}$ Another analysis demonstrated that population-based carrier screening for FXS meets the criteria for a screening test. ${ }^{22}$ In a pilot program in Finland, $85 \%$ of pregnant women offered FXS carrier testing requested the testing. ${ }^{23}$

There have been currently no available techniques to perform accurate, automated, high-throughput carrier detection for FXS. Determining a woman's carrier status require analysis by both polymerase chain reaction (PCR) and Southern blot. This is because expanded alleles of $>100$ repeats are resistant to amplification using PCR. Southern blots are manual, timeconsuming, and labor intensive, require relatively large amounts $(1 \mu \mathrm{g})$ of intact DNA, and are not amenable to the automation and high throughput necessary for populationbased carrier screening. Although there have been reports of other potential screening methods, ${ }^{24,25}$ none was sufficiently accurate, robust, and automatable to fulfill the requirements of population-based carrier screening.

In this report, we describe the development of an accurate, automated, high-throughput technique suitable for population-based carrier screening for FXS. This is a two-step process: an initial PCR assay followed by a new methodology we call capillary Southern analysis (CSA). This technique allows automated, high-throughput carrier screening to be performed for FXS at a reasonable cost. To date, this new assay has shown $100 \%$ sensitivity and at least $99.5 \%$ specificity for carrier detection of FXS when compared with the gold standard of PCR followed by Southern blot analysis.

\section{METHODS AND MATERIALS}

\section{General considerations}

All procedures in this assay are carried out by automation. All reaction setups are performed using automated liquid handlers (BioMek-2000, Beckman-Coulter, Fullerton, CA).

\section{Genomic DNA extraction from whole blood for PCR/Southern blot analysis}

Three milliliters of whole blood were extracted on a Gentra Autopure LS (Minneapolis, MN) exactly as described by the manufacturer.

\section{Genomic DNA extraction from whole blood for PCR/CSA}

For the automated PCR/CSA assay, genomic DNA was extracted from $150 \mu \mathrm{L}$ whole blood collected in ethylenediamine tetraacetic acid (EDTA) anticoagulated blood collection vacuum tubes using an X-tractor Gene (Corbett Life Science, Mortlake, NSW, Australia) according to manufacturer's Whole Blood DNA Extraction Protocol. The final elution was carried out in $100 \mu \mathrm{L}$ of buffer to consistently yield concentrations of $50-100 \mathrm{ng} / \mu \mathrm{L}$. For Southern blots, DNA was isolated using the Autopure LS according to manufacturer's protocol.

\section{Multiplex PCR for androgen receptor, FMR1, and amelogenin}

This multiplex PCR amplifies the FMR1 region that includes the CGG triplet repeats, a region of the amelogenin gene that yields different fragment sizes from the $\mathrm{X}$ and $\mathrm{Y}$ chromosomes for gender determination, and a polymorphic region of the androgen insensitivity gene used as an internal control. ${ }^{26}$ The PCR mix consists of $3.3 \mu \mathrm{M}$ of each of following primers: AMLF2 primer, FAM-labeled, 5' AGTACTTGACCACCTCCTGATCTACAAGG 3'; AMLR2 primer, 5' TTTTTAACAGTTTACTTGCTGATAAAACTCAYCCC $3^{\prime} ; 3.3 \mu \mathrm{M}$ of each of following primers: FX-5F primer, FAM-labeled, 5' CTCAGCTCCGTTTCGGTTTCACTTCCGGT 3'; FX-3F primer, 5' AGCCCCGCACTTCCACCACCAGCTCCTCCA 3'; AR-5F primer, 
HEX-labeled, 5' ACCAGGTAGCCTGTGGGGCCTCTACGATGGGC 3'; AR-R2 primer, 5' GCTTTCCAGAATCTGTTCCAGAGCGTGCGCGA $3^{\prime}$. These primers were added to the PCR master mix consisting of $1 X$ Qiagen Standard PCR buffer, $0.4 \mathrm{mM}$ $\mathrm{MgCl}_{2}, 2 \%$ dimethyl sulfoxide (DMSO), 1X Qiagen Q Solution, $0.2 \mathrm{mM}$ deoxyribonucleotide triphosphate (dNTP), and 0.25 unit Qiagen Taq DNA polymerase (Qiagen, Valencia, CA), 0.5 unit $P f u$ DNA Polymerase (Strategene, La Jolla, CA). One microliter of isolated DNA solution was added to $10 \mu \mathrm{L}$ of the multiplex primer mix to a final volume of $11 \mu \mathrm{L}$. The target DNA was amplified after heat initiation at $95^{\circ} \mathrm{C}$ for 6 minutes followed by 32 cycles of $95^{\circ} \mathrm{C}$ for 1 minute, $60^{\circ} \mathrm{C}$ for 2 minutes, $75^{\circ} \mathrm{C}$ for 5 minutes, and finally the amplified products were extended at $75^{\circ} \mathrm{C}$ for 15 minutes.

The PCR fragments were analyzed on an ABI 3100 automated DNA sequencer (Applied Biosystems, Foster City, CA), and fragment analysis was accomplished with ABI GeneScan V3.7 and Genotyper V3.7 software (Applied Biosystems).

\section{Capillary electrophoresis and fraction collection of FMR1 gene fragments}

Genomic DNA extracted from whole blood was digested for 16 hours at $37^{\circ} \mathrm{C}$ with restriction enzymes $B l p \mathrm{I}$ and $M l y \mathrm{I}$ (New England BioLabs, Ipswich, MA). After incubation, the restriction fragments were either pressure injected or vacuum injected onto a P/ACEMDQ capillary electrophoresis system with a UV/Vis Detector (Beckman Coulter, Fullerton, CA). Undenatured double-stranded DNA was separated at an electric field strength of $100 \mathrm{~V} / \mathrm{cm}$, in $1 X$ Tris-Borate EDTA buffer (90 mM Tris-borate, 2 mM EDTA, $\mathrm{pH}$ 8.3). Capillary temperature was maintained at $25^{\circ} \mathrm{C}$. Four fractions were collected into 0.1X TBE buffer ( $9 \mathrm{mM}$ Tris-borate, $0.2 \mathrm{mM}$ EDTA, $\mathrm{pH}$ 8.3). The initial fraction consisted of molecular weights between 400 and $600 \mathrm{bp}$, the second fraction included molecular weights of between 600 and $800 \mathrm{bp}$, the third fraction included molecular weights of 800-1000 bp, and the fourth fraction included molecular weights of 1000-8000 bp. No internal control for incomplete restriction digestion is needed because failure of either enzyme to cleave in FMR1 would result in a fragment too large to be collected in any fraction.

\section{Amplification of collected fractions by fluorescent PCR}

All collected fractions were then subjected to restriction enzyme digestion with BmtI (New England BioLabs) according to manufacturer's procedure to liberate the flanking fragment to be amplified from the CGG repeats. The multiplex PCR mixture contains primers to amplify a flanking region $3^{\prime}$ to the CGG repeats plus internal controls for each of Fractions 2-4. An additional primer pair is present as an internal control for incomplete restriction digestion by Mly1. After complete digestion, no amplicon will be detected from this primer pair, whereas if incomplete digestion has occurred, an additional amplicon will appear in fraction 3. Five microliters of each digested fraction was transferred to 96 -well plates containing $20 \mu \mathrm{L}$ of PCR mix in each well. This PCR mix consists of $1 \mathrm{X}$ Qiagen Standard PCR buffer, $1.5 \mathrm{mM} \mathrm{MgCl}_{2}, 5 \%$ dimethyl sulfoxide, $100 \mathrm{mM} \mathrm{KCl}$,
$0.2 \mathrm{mM}$ dNTP, $1 \mu \mathrm{M}$ of each of following primers: FMR1F2 primer, FAM-labeled, 5' GATGGAGGAGCTGGTGGTGG 3'; FMR1R2 primer, 5' GGAAGGGCGAAGATGGGG 3', $0.01 \mu \mathrm{M}$ of each of following primers: BlpIF primer, HEX-labeled, $5^{\prime}$ AGTGTTTAGAAGGAAAAGGCTGAGC 3'; BlpIR primer, 5' GCCCAAAGTTTCATAGGTAGCAAA $3^{\prime}$; lgctrlF primer, FAM-labeled, 5' AGGTTTGAGTGTATCGCCTGATAGA 3'; lgctrlR primer, 5' TGAGTTTCATGTTTGCTCTTGCTC 3'; F2ctrl1F primer, HEXlabeled, 5' CTGAATTTGTTTGGTTTGATGATGC 3'; F2ctrl1R primer, 5' CCTGTGTTATCTGTGCCCATTTTAA 3'; F3ctrl3F primer, FAM-labeled, 5' ATCTGGGTCTGAATAATGTGAGGAG 3'; F3ctrl3R primer, 5' CCTAACTTTCATTCTTGTCACCCTT $3^{\prime} ; 2.5$ units of HotStart Taq DNA polymerase (Qiagen Inc.). The targeted DNA was amplified after heat initiation at $95^{\circ} \mathrm{C}$ for 15 minutes followed by 33 cycles at $95^{\circ} \mathrm{C}$ for 30 seconds, at $55^{\circ} \mathrm{C}$ for 30 seconds, at $72^{\circ} \mathrm{C}$ for 1 minute, and finally the amplified products were extended at $72^{\circ} \mathrm{C}$ for 10 minutes. The final PCR products were then analyzed on a 3100 Prism Genetics Analyzer (Applied Biosystems) using Genescan-350 ROX size standard (Applied Biosystems).

\section{Work flow}

In terms of assay flow, blood is aliquotted into 96-well microtiter plates using an automated liquid handler. After DNA isolation, the samples are aliquotted into two separate duplicate plates. The PCR and automated analysis are performed in one plate, whereas restriction enzymes are added to the second plate and an overnight digestion is performed. After review, all males with a normal allele and all females heterozygous for two normal alleles are reported as normal. The following day, the automated analysis of the PCR results is communicated to the capillary instrument, and the appropriate samples (homozygous females and males with no FMR1 amplification) are injected into the capillary using the instructions from the sequencing instrument. After fraction collection, multiplex PCR and analysis on the capillary sequencer are performed. All patients with normal CSA results are reported as normal. For patients in whom the CSA indicates an expanded allele, the original blood tube is retrieved and a Southern blot is performed to confirm the results.

\section{RESULTS}

We have been performing FXS testing using a combination of PCR and Southern blot analysis since 1992. ${ }^{26}$ A reasonable strategy for population-based carrier screening for FXS could use an initial screen with a PCR-based assay. Any woman with two different alleles could be assumed to be a noncarrier. Any woman heterozygous for a "gray zone" or premutation allele that amplified during the PCR reaction could also be reported without further analysis, although often a Southern blot could be performed to screen for mosaicism. However, if in the PCR, a woman appears to be homozygous at the FMR1 site, until now, there has been no reliable method other than a Southern blot to determine whether she is a true homozygote for two normal alleles or a heterozygote for a normal allele and an 

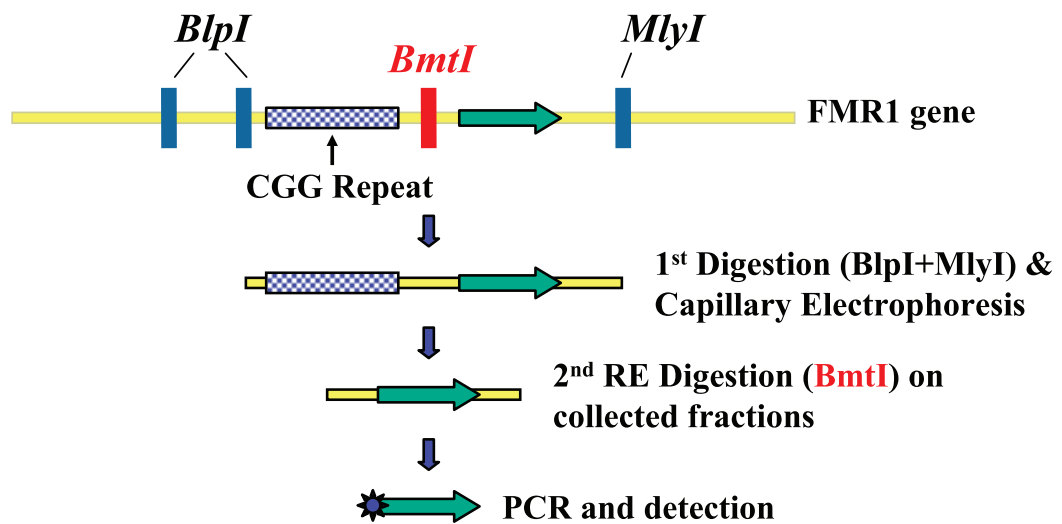

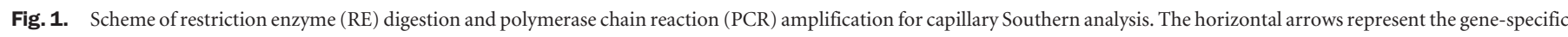
PCR amplicon.

expanded allele that could not be amplified by the PCR. In our experience, 5677 (24\%) of 23,310 women determined to be noncarriers of FXS by PCR and Southern blot analysis were homozygous at the FMR1 locus in the PCR analysis. This number is not unexpected because in our series of more than 119,000 FXS tests, $51 \%$ of normal alleles were 29,30 , or 31 repeats. ${ }^{27}$ Because these alleles are difficult to separate from each other on the capillary, an apparent homozygosity of $24 \%$ is reasonable. Using standard FMR1 PCR analysis, therefore, almost one fourth of women screened would require a Southern blot to complete their analysis. Using currently available techniques, if population-based carrier screening were introduced in the United States with its approximately 4.4 million births per year, the number of Southern blots necessary to screen the population would be prohibitively high.

We initially attempted to perform quantitative analysis on the size of the FMR1 peak and compare it with internal controls but found this technique to miss a significant percentage of known carriers. Therefore, we developed a new technique, CSA, as a reflex for the $24 \%$ of women who will be homozygous in our PCR assay.

The initial PCR is a multiplex assay that includes an amelogenin internal control to confirm gender and the androgen receptor gene as a second internal control. These internal controls prevent false-positive results for male samples due to PCR failure and confirm the gender of the patient sample. This initial PCR reaction is analyzed on the automated capillary DNA sequencer that is only capable of resolving fragments up to approximately 100 repeats. The PCR itself is only capable of amplifying fragments up to 100 repeats, so 100 repeats is the upper limit of detection for the initial PCR in our scheme. If a woman is found to have a premutation allele by PCR, a Southern blot is performed to investigate the possibility of mosaicism for a larger expanded allele.

Samples from women who are homozygous for a normal FMR1 allele are subsequently analyzed by CSA to identify the samples truly homozygous for a normal allele and those who are heterozygous for a normal and an expanded allele. The details of the CSA are described in the Methods and Materials section and the scheme is shown in Figure 1.
Southern blotting and CSA are very similar in principle. In both techniques, restriction enzymes digest the DNA into fragments of varying sizes. After digestion, a size fractionation is performed. In Southern blotting, the size fractionation is performed on an agarose slab gel; in CSA, it is performed on a capillary. In Southern blots, smaller fragments travel further in the gel than longer fragments at a given electrophoresis time. In CSA, smaller fragments travel to the end of the column and are eluted off the end of the column and into the fraction collector at earlier times (and therefore earlier fractions) than longer fragments. In Southern blots, all fragments of a similar size will migrate to the same physical point in the gel, whereas in CSA, all fragments of similar size are eluted from the end of the gel at a similar time and are therefore collected in the same fraction. In a Southern blot, the location of the gene specific sequence is identified by transferring the DNA to a membrane and hybridizing the membrane with a labeled gene-specific probe. In CSA, the presence or absence of a specific gene sequence is determined by gene-specific PCR of the collected fragments. In Southern blots, the size of the specific fragment is determined by the position of migration in the gel. In CSA, the size of the gene-specific fragments are determined by the fraction or fractions containing the gene-specific PCR product. Because of the nature of Southern blotting versus PCR, CSA is able to detect as few as 60 molecules of gene-specific material, whereas Southern blotting requires at least 100,000 copies of gene-specific material.

Initially, a restriction digestion is performed with two restriction enzymes, $B l p 1$ that cleaves $5^{\prime}$ to the CGG repeat region and Mly 1 that cleaves $3^{\prime}$ to the repeat region (Fig. 1). The next adjacent $M l y 1$ sites are 1119 bp upstream ( $3^{\prime}$ ) and $1334 \mathrm{bp}$ downstream $\left(5^{\prime}\right)$, respectively. These enzymes were chosen using a bioinformatics approach. We required enzymes that would cleave the gene into sizes capable of being resolved by the capillary. No single enzyme cleavage was appropriate and neither enzyme could be inhibited by methylation. After restriction digestion, the fragments are size fractionated on a capillary electrophoresis device that has a fraction collector. Four fractions are collected as follows: fraction 1: 6-63 repeats, 
High-throughput technique for FXS carrier screening

fraction 2: 64-140 repeats, fraction 3: 141-220 repeats, fraction 4: 221-2000 repeats.

For each of fractions 2-4, an invariant restriction fragment was chosen as an internal control for gel separation and PCR success. Selecting appropriate fragments required a bioinformatics approach because restriction digestion with $B l p 1$ and $M l y 1$ of the human genome will generate approximately 3 million digested fragments. Bioinformatics tools allow a prediction of the results of this restriction digestion in silico. Fragments predicted to be of the appropriate size were then subjected to further scrutiny in terms of guanidine/cytidine content, uniqueness in the genome, and known polymorphisms before selecting candidate sequences to use as internal controls.

No control for incomplete $M l y 1$ digestion is needed because failure of restriction digestion leads to fragments too large to be collected in any of the fractions. An internal control for incomplete digestion $B l p 1$ is required and is incorporated into the postfractionation multiplex PCR (see Methods and Materials). Because all samples are subjected to PCR initially, alleles containing up to 100 CGG repeats will be identified by PCR. Therefore, a sample homozygous for a normal allele and having material only in fraction 1 will be from a noncarrier female.

After fractionation, the used gel is discarded and the capillary is filled with a new gel matrix to prevent any carryover contamination. After electrophoresis, each fraction is subjected to a second restriction digestion, this time with the methylation-insensitive enzyme Bmt1. This liberates the fragment to be amplified from the CGG repeats, increasing PCR robustness and detection of as few as 60 molecules of DNA. We refer to this restriction digestion as liberating PCR because it frees the PCR amplicon from its anchor of CGG repeats. Liberating PCR increases the sensitivity of the PCR by 6- to 10fold. We assume that the CGG repeats either do not melt at normal PCR conditions or contain a secondary structure that causes the attached DNA to reanneal more rapidly than liberated sequences, thus inhibiting the efficiency of amplification. Without liberating PCR, more DNA had to be added to the capillary to get reliable detection, and this amount of DNA overloaded the separating capacity of the capillary and fragments "leaked" into inappropriate fractions. Therefore, liberating PCR is a necessary step to enable CSA to work.

After restriction digestion, a multiplex PCR is performed containing primers specific for the $3^{\prime}$ flanking sequence (Fig. 1 ), and internal controls for fractions 2,3 , and 4 . The internal controls ensure that the size fractionation on the capillary and the PCR have performed acceptably in all fractions. The PCR products are analyzed on an automated DNA sequencer with automated allele calling (see Methods and Materials). Analysis of the data is performed automatically. If all internal controls have performed acceptably, interpretation can be made by using the scheme shown in Table 1.

CSA is meant as a first-level screening test, and in the current iteration is not capable of assessing methylation status. Only samples that are homozygous on PCR and positive (having amplified material in fractions 2-4) are considered positive
Table 1

Interpretation of capillary Southern analysis (gender has been previously determined by polymerase chain reaction)

\begin{tabular}{|c|c|c|c|c|}
\hline Interpretation & Fraction 1 & Fraction 2 & Fraction 3 & Fraction 4 \\
\hline Female, noncarrier & + & - & - & - \\
\hline Female, premutation carrier & + & + & - & - \\
\hline $\begin{array}{l}\text { Female, carrier, either full or } \\
\text { premutation }^{a}\end{array}$ & + & - & + & - \\
\hline Female, full mutation carrier & + & - & - & + \\
\hline Normal male & + & - & - & - \\
\hline Male, premutation & - & + & - & - \\
\hline Male, full or premutation ${ }^{a}$ & - & - & + & - \\
\hline Male, full mutation (affected) & - & - & - & + \\
\hline
\end{tabular}

${ }^{a}$ Southern blot required to determine methylation status and size of expansion.

and reflexed to Southern blot for methylation analysis. Because the carrier frequency for FXS in the U.S. population will probably be no more than $1 \%$, the number of Southern blots necessary will be reduced by $99 \%$, thus making the number of Southern blots manageable in a population-based screening test. Work is progressing to perform methylation analysis using CSA so that Southern blots can be eliminated completely.

This method is capable of detecting premutation and affected males and could be used as an initial screen for population-based screening for affected males, but the purpose of this study was to determine its appropriateness for populationbased carrier detection. The male controls were used to validate the technique for potential future uses in the diagnosis of FXTAS and FXS in males.

Once the assay was fully developed, we performed blinded testing on samples from 19 heterozygous females who have a normal allele and an affected allele (affected females) and 8 affected males. These diagnoses were previously made by Southern blot analysis using methylation-specific enzymes. ${ }^{26}$ Sometimes expanded alleles will demonstrate a "smear" on Southern blot. In such cases, the expanded allele is described by the size range of the smear. Table 2 shows the allele sizes of the affected females and affected males that were part of this series.

Affected alleles from between 200 and 1450 CGG repeats were detected, and all samples were correctly identified in this series. There was no difficulty making the correct diagnosis in samples demonstrating mosaicism on Southern blot. The single ambiguous result was a male with 200 CGG repeats by Southern blot that had an FMR1 amplicon in fraction 3. Samples positive only in fraction 3 could be either premutation alleles or small affected alleles. All such cases as this will require Southern Blot analysis to examine the methylation status as well as the size of the expanded allele.

We also blinded samples from four females diagnosed as heterozygous for a normal allele and an unmethylated premutation allele consisting of 100-200 CGG repeats. All cases were correctly diagnosed by the presence of amplified product in fraction 3. 
Table 2

Patient samples with affected alleles used in assay development

\begin{tabular}{|c|c|c|}
\hline Final diagnosis & $\begin{array}{l}\text { Southern } \\
\text { blot result }\end{array}$ & CSA result \\
\hline Affected female & $35 / 250-600$ & Normal/full expansion \\
\hline Affected female & $29 / 400$ & Normal/full expansion \\
\hline Affected female & $30 / 260$ & Normal/full expansion \\
\hline Affected female & $29 / 200-450$ & Normal/full expansion \\
\hline Affected female & $30 / 500$ & Normal/full expansion \\
\hline Affected female & $29 / 350$ & Normal/full expansion \\
\hline Affected female & $31 / 250-750$ & Normal/full expansion \\
\hline Affected female & $28 / 600$ & Normal/full expansion \\
\hline Affected female & 29/400-900 & Normal/full expansion \\
\hline Affected female & $30 / 600$ & Normal/full expansion \\
\hline Affected female & $29 / 600$ & Normal/full expansion \\
\hline Affected female & $29 / 300-750$ & Normal/full expansion \\
\hline Affected female & $30 / 500$ & Normal/full expansion \\
\hline Affected female & $30 / 450$ & Normal/full expansion \\
\hline Affected female & $26 / 850$ & Normal/full expansion \\
\hline Affected female & $30 / 500-1450$ & Normal/full expansion \\
\hline Affected female & $30 / 350$ & Normal/full expansion \\
\hline Affected female & $29 / 950$ & Normal/full expansion \\
\hline Affected female & $33 / 800$ & Normal/full expansion \\
\hline Affected male & $400-1000$ & Full expansion \\
\hline Affected male & $200-450$ & Full expansion \\
\hline Affected male & $400-1300$ & Full expansion \\
\hline Affected male & $200-950$ & $\begin{array}{l}\text { Mosaic full expansion } \\
\quad \text { (Fractions } 3 \text { and } 4 \text { positive) }\end{array}$ \\
\hline Affected male & 350 & Full expansion \\
\hline Affected male & 200 & Premutation or full expansion \\
\hline Affected male & 300 & Full expansion \\
\hline Affected male & 300 & Full expansion \\
\hline
\end{tabular}

CSA, capillary Southern analysis.

Once we were confident in the ability of this scheme to correctly identify carriers for FXS, we prepared three validation plates containing 14 females homozygous for two normal alleles, 14 females heterozygous for a normal allele, and an affected allele, three males hemizygous for an affected allele and four females heterozygous for a normal and a premutation allele. Each sample was analyzed in triplicate in each plate. The identity of the samples was blinded, the positions randomized in each plate. Two different laboratory personnel using two different capillary instruments performed analysis at 1-week intervals. The CSA was $100 \%$ sensitive and $100 \%$ specific in identifying patients with expanding $F M R 1$ alleles. There was no intraor interassay variability in terms of genotype assignment.

CSA was also quite robust, with 101 of 105 (96\%) successful analyses. Two of the failed reactions occurred in the same pa- tient, indicating that there might be a low DNA concentration in that sample. Thirty-four of 35 (97\%) patients were successfully genotyped after the first analysis, and one patient sample yielded a result in only the third experiment. These data demonstrate that multiplex PCR followed by CSA is capable of identifying carriers for FXS in a highly sensitive and specific manner.

The initial studies described above were all performed on DNA prepared for Southern blot analysis on a Gentra Autopure robotic system as described in Methods and Materials. This method of DNA extraction is not suitable for high throughput screening as only 16 samples can be processed in a 2 hour time frame and reagents and equipment are costly. The $\mathrm{PCR} / \mathrm{CSA}$ technique is designed to be performed on samples prepared by the Korbett system (see Methods and Materials), which processes 96 samples at a time with a low reagent and equipment cost.

To test the ability of PCR/CSA to detect FXS carriers in a normal laboratory work flow using Korbett extracted DNA, we performed a parallel study of samples submitted for routine FXS diagnostic and carrier testing. When the physician provided more than one tube of blood, we stripped the second tube of identifiers and used it to perform the automated PCR/ CSA exactly as described in Methods and Materials. In this series of 995 female patients, there were six premutation females $(0.6 \%)$ and seven full mutation females $(0.7 \%)$. All 13 carriers were identified by CSA leading to a sensitivity of $100 \%$. There was a single patient who was interpreted as negative by the standard PCR/Southern blot assay who appeared to be a premutation carrier by the PCR/CSA assay. It is possible that this patient is mosaic for a premutation allele or that this represents a false-positive result. Because the sample had been anonymized, it was not possible to review the Southern blot data or to retest the sample. Because our testing protocol would involve confirming the CSA with a Southern blot, this would not have resulted in reporting a false-positive result. Assuming this result is a false positive, the specificity of PCR/ CSA is $99.5 \%$.

We also performed PCR/CSA on 557 male patients in this series. There was one premutation male and five affected males. PCR/CSA detected all premutation and affected patients with no false-positive results on the 551 unaffected males.

Figures 2-4 are representative results from this comparison series of 995 females and 557 males. Figure 2 is a noncarrier female. An FMR1 product is only seen in fraction 1, and all internal controls are present in the appropriate fractions. A mosaic-affected female is shown in Figure 3, with FMR1-specific amplicons present in fractions 1 and 4 with all internal controls present. Southern blot analysis demonstrated this patient to be mosaic for an expansion of 400-650 CGG repeats. A mosaic female is presented in Figure 4 with FMR1specific amplicons present in fractions $2-4$. Southern analysis revealed this woman to be mosaic for an allele with 200-350 CGG repeats. 


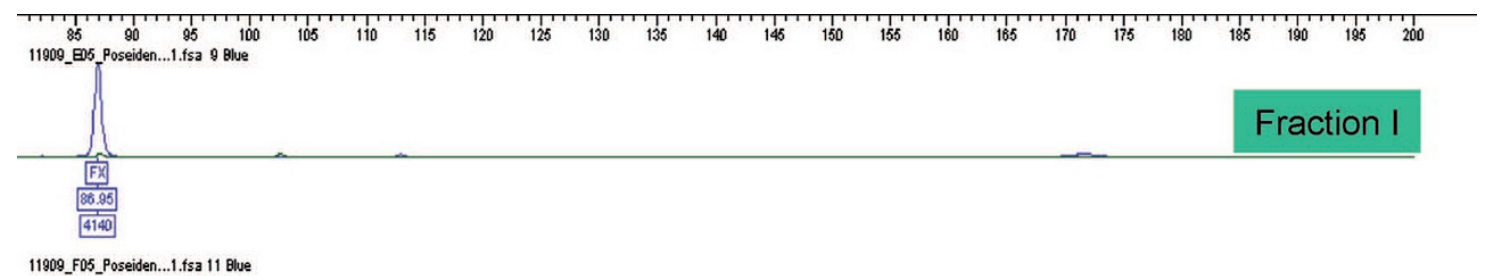

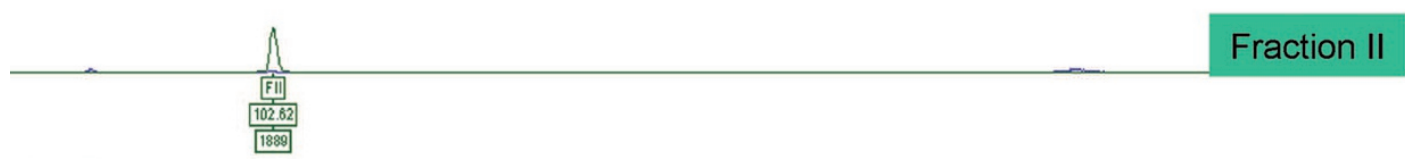

11909_C05_Poseiden...1.fsa 13 Blue

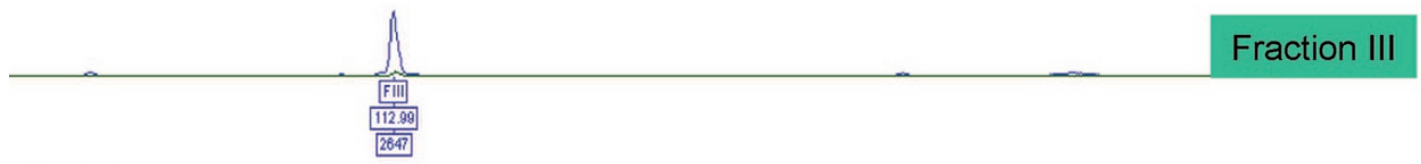

11909_H05_Poseiden...1.tsa 15 Blue

Fraction IV

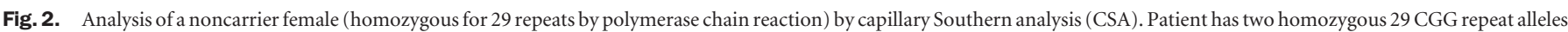

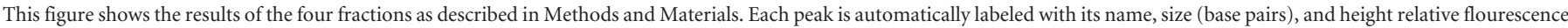

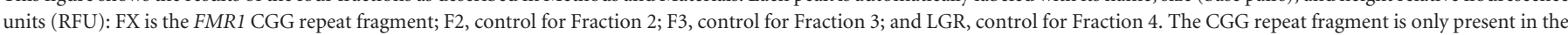
first fraction, and all three control fragments are present in their proper fractions indicating a noncarrier female.
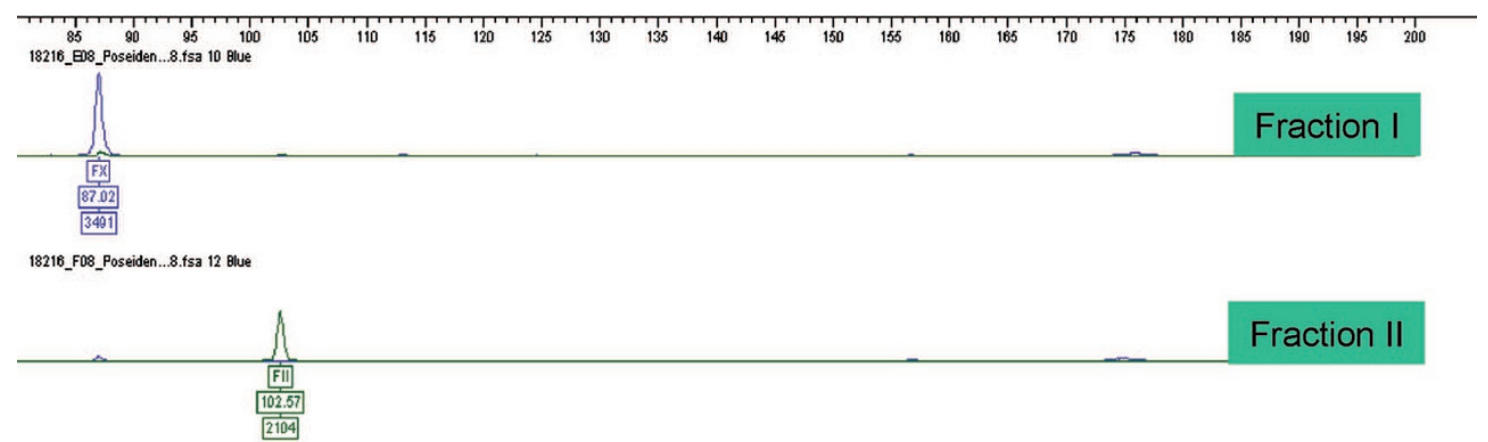

18216_G08_Poseiden...8.tsa 14 Blue

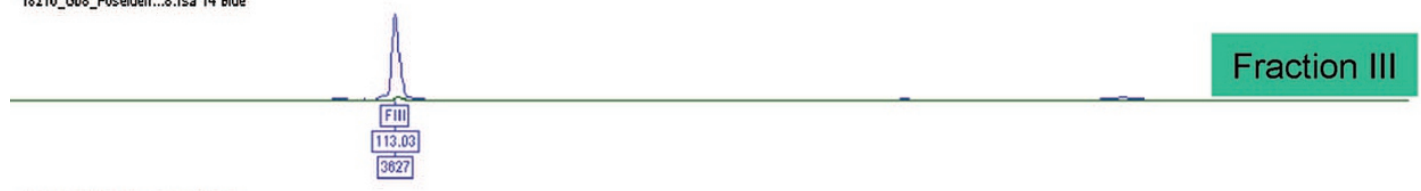

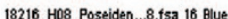

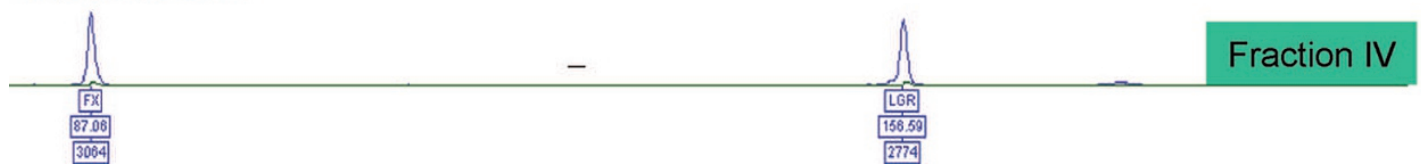

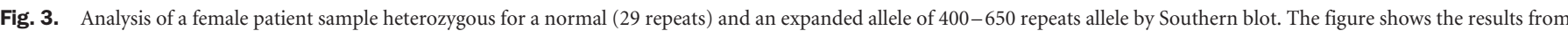

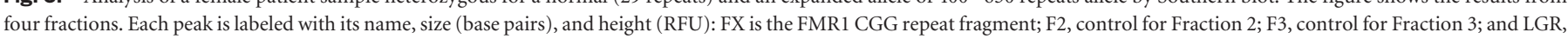
control for Fraction 4. The CGG repeat fragment is present in both the first and fourth fractions; all three control fragments are present in their proper fractions.

\section{DISCUSSION}

We have developed a novel, rapid, automated, highthroughput assay capable of detecting female FXS carriers with $100 \%$ sensitivity and at least $99.5 \%$ specificity and premutation and affected males with $100 \%$ sensitivity and specificity. This assay detects both premutation and full mutation carriers. Theoretically, this assay will detect expanded alleles of up to 2000 CGG repeats, although we do not have any nonmosaic controls with this large an expansion to demonstrate this. In our testing paradigm, we would initially confirm all positive 

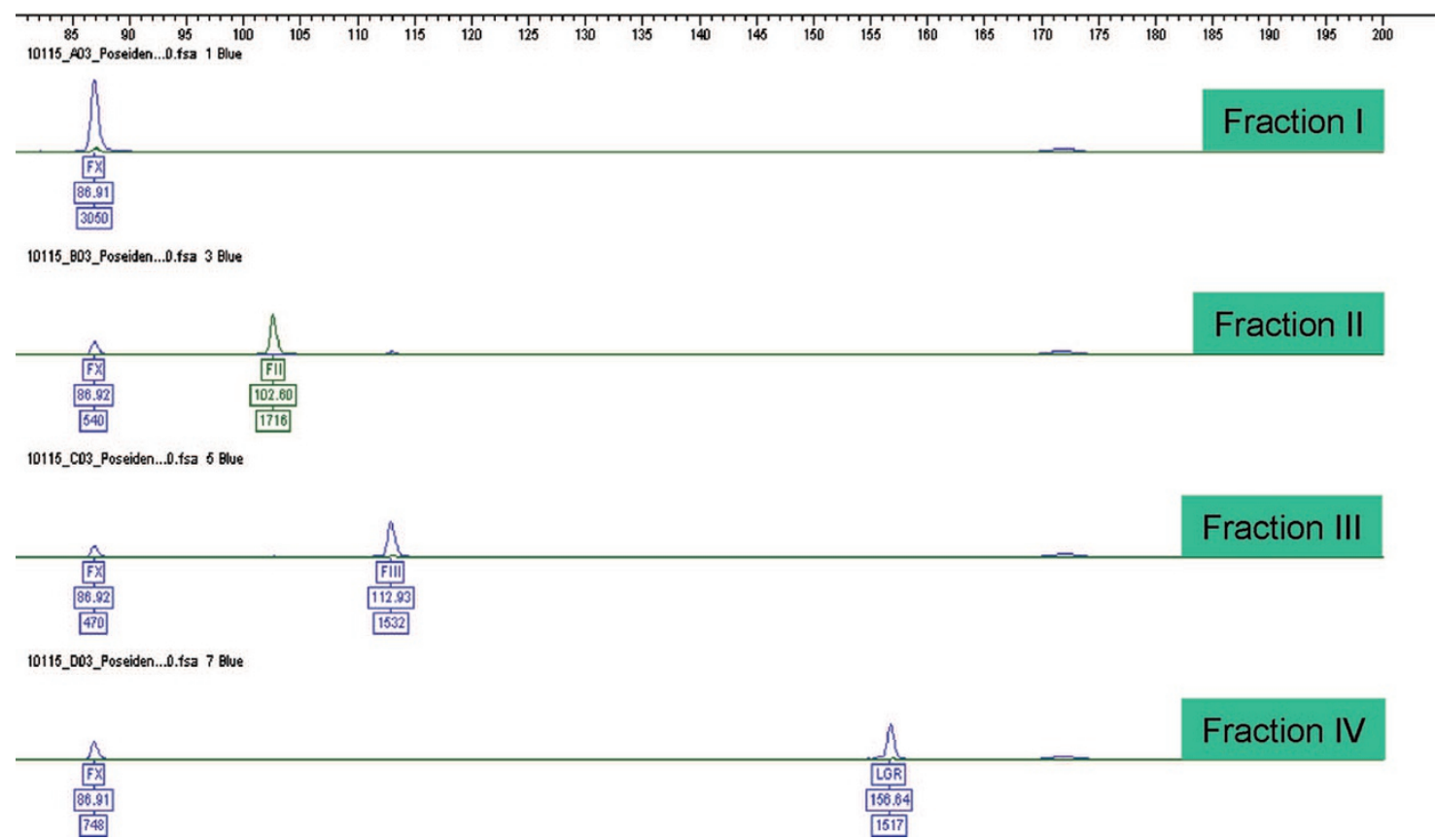

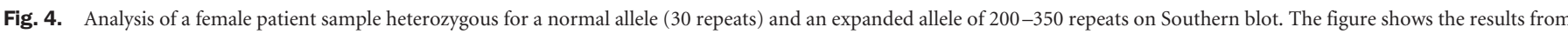

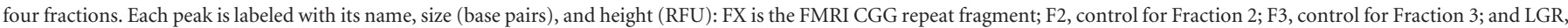
control for Fraction 4. The CGG repeat fragment is present in all fractions; all three control fragments are present in their proper fractions.

CSA analyses with a Southern blot. In our series, this would mean only $1.3 \%$ of female samples would require Southern blot analysis, an approximate 20 -fold reduction in the need for Southern blots with respect to using PCR alone.

Every step of the assay is done in 96-well microtiter plates and performed by automated systems, including liquid handlers, DNA purification instruments, automated capillary electrophoresis instruments, and automated DNA sequencers. The analysis is also automated as is the communication between the initial PCR results to the CSA instrument to inform the capillary what samples require CSA. Therefore, this technology will allow population-based carrier detection for FXS and potentially newborn screening for FXS to be performed in a cost-effective manner.

A complete CSA system consists of a DNA purification system, liquid-handling station, capillary electrophoresis apparatus, thermocycler, and automated DNA sequencer. This system can easily handle two plates (approximately 180 samples) in an 8-hour shift in 2 days. Therefore, with continuous flow processes, one system and a single laboratory technologist could handle 65,700 patients per year using a single 8 -hour shift. Capacity could be added by simply increasing the number of complete systems in operation.

Although an exhaustive financial analysis has not yet been completed, the cost to perform population-based carrier screening by CSA appears to be on the same order of magnitude as for cystic fibrosis, Tay-Sachs disease, familial dysautonomia, and Canavan disease. There have been American College of Medical Genetics recommendations for population-based carrier detection for cystic fibrosis for Caucasians and a combination of cystic fibrosis, Tay-Sachs disease, familial dysautonomia, and Canavan disease in individuals of AJ descent.

We are also developing an alternative to Southern blots using CSA by collecting more fractions and using a methylationsensitive restriction enzyme. CSA might also be able to replace Southern blots for other applications, including methylation analysis, and could also be used for epigenetic assays involving methylation assessment.

We describe a new technology, CSA, that is capable of performing population-based FXS carrier screening or newborn screening in an automated, high-throughput, cost-efficient manner. This technology may also replace Southern blot analysis in other applications.

We are committed to making this technology widely available to facilitate population-based carrier detection for FXS. Inquiries regarding access to this technology should addressed to Nick Conti, Quest Diagnostics Vice President of Licensing and Alliances, at the following e-mail address: nicholas.j.conti@ questdiagnostics.com.

\section{References}

1. Oberle I, Rousseau F, Heitz D, Kretz D, et al. Instability of a 550-Base Pair DNA segment and abnormal methylation pattern in fragile X syndrome. Science 1991;252: 1097-1102.

2. Crawford DC, Meadows KL, Newman JL, Taft LF, et al. Prevalence and phenotype consequence of FRAXA and FRAXE alleles in a large, ethnically diverse, special education-needs population. J Am Hum Genet 1999;64:495-507.

3. Moore SJ, Strain L, Cole GF, Miedzybrodzka Z, et al. Fragile X syndrome with FMR1 and FMR2 deletion. J Med Genet 1999;36:565-566.

4. Parvari R, Mumm S, Galil A, Manor E, et al. Deletion of $8.5 \mathrm{Mb}$, including the FMR1 gene, in a male with the Fragile X syndrome phenotype and overgrowth. Am J Med Genet 1999;83:302-307. 
5. Quan F, Zonana J, Gunter K, Peterson K, et al. An atypical case of fragile X syndrome caused by a deletion that includes the FMR1 gene. Am J Hum Genet 1995;56:1042_ 1051.

6. Wolff DJ, Gustashaw KM, Zurcher V, Ko L, et al. Deletions in Xq26.3-q27.3 including FMR1 result in a severe phenotype in a male and variable phenotypes in females depending upon the X inactivation pattern. Hum Genet 1997;100: 256-262.

7. Hammond LS, Macias MM, Tarleton JC, Pai GS. Fragile X syndrome and deletions in FMR1: New case and review of the literature. Am J Med Genet 1997;72:430-434.

8. Grønskov K, Hallberg A, Brøndom-Nielsen K. Mutational analysis of the FMR1 gene in 118 mentally retarded males suspected of fragile $\mathrm{X}$ syndrome: absence of prevalent mutations. Hum Genet 1998;102:440-445.

9. Maddalena A, Richard CS, McGinniss MJ, Brothman A, et al. Technical standards and guidelines for fragile X. Genet Med 2001;3:200-205.

10. Nolin SL, Brown WT, Glicksman A, Houck GE Jr, et al. Expansion of the Fragile X CGG repeat in females with premutation or intermediate alleles. Am J Hum Genet 2003;72:454-464.

11. Sullivan AK, Marcus M, Epstein MP, Allen EG, et al. Association of FMR1 repeat size with ovarian dysfunction. Hum Reprod 2005;20:402-412.

12. Bretherick KL, Fluker MR, Robinson WP. FMR1 repeat sizes in the gray zone and high end of the normal range are associated with premature ovarian failure. Hum Genet 2005; 117:376-382.

13. Hagerman PJ, Hagerman RJ. The fragile X premutation: a maturing perspective. Am J Hum Genet 2004;74:805-816.

14. Jacquemont S, Hagerman RJ, Leehey MA, Hall DA, et al. Penetrance of the fragile $\mathrm{X}$-associated tremor/ataxia syndrome in a premutation carrier population. JAMA 2004;291:460-469.

15. Pesso R, Berkenstadt M, Cuckle H, Gak E, et al. Screening for fragile X syndrome in women of reproductive age. Prenat Diagn 2000;20:611-614.

16. Toledano-Alhadef H, Basel-Vanagaite L, Magal N, Davidov B, et al. Fragile-X carrier screening and the prevalence of premutation and full-mutation carriers in Israel. Am J Hum Genet 2001;69:351-360.
17. Rousseau F, Rouillard P, Morel ML, Khandijan EW, et al. Prevalence of carriers of premutation-size alleles of the FMR1 gene-and implications for the population genetics of the fragile X syndrome. Am J Hum Genet 1995;57:10061018.

18. Grody WW, Cutting GR, Klinger KW, Richards CS, et al. Laboratory standards and guidelines for population-based cystic fibrosis carrier screening. Genet Med 2001;3: 149-154.

19. American College of Obstetrics and Gynecology and American College of Medical Genetics. Preconception and Prenatal Carrier Screening for Cystic Fibrosis: Clinical and Laboratory Guidelines. Washington, DC: American College of Obstetricians and Gynecologists, 2001.

20. American College of Obstetrics and Gynecology Committee on Genetics. Prenatal and preconceptional carrier screening for genetic diseases in individuals of Eastern European Jewish descent. Obstet Gynecol 2004;104: 425-428.

21. Musci TJ, Caughey AB. Cost-effectiveness of prenatal population based fragile X carrier screening. Obstet Gynecol 2005;192:1905-1915.

22. Palomaki GE. Population based prenatal screening for the fragile X syndrome. J Med Screen 1994;1:65-72.

23. Ryynänen M, Heinonen S, Makkonen M, Kajanoja E, et al. Feasibility and acceptance of screening for fragile X mutations in low-risk pregnancies. Eur J Hum Genet 1999;7:212-216.

24. Saluto A, Brussino A, Tassone F, Arduino C, et al. An enhanced polymerase chain reaction assay to detect pre- and full mutation alleles of the fragile $\mathrm{X}$ mental retardation 1 gene. J Mol Diagn 2005;7:1-8.

25. Zhou Y, Law H-Y, Boehm, CD, Yoon C-S, et al. Robust fragile X (CGG) nenotype classification using a methylation specific triple PCR assay. J Med Genet 2004;41:e45.

26. White BJ, Ayad M, Fraser A, Entwistle T, et al. A 6-year experience demonstrates the utility of screening for both cytogenetic and FMR-1 abnormalities in patients with mental retardation. Genet Test 1999;3:291-296.

27. Strom CM, Crossley B, Redman JB, Buller A, et al. Molecular testing for fragile X syndrome: lessons learned from 119,232 tests performed in a clinical laboratory. Genet Med, 2007;9:46-51. 